\title{
Plasticity and Thickness Effect
}

\section{ANQUEZ}

Office National d'Etudes et de Recherches Aérospatiales, BP 72-92322 Châtillon Cedex, France

\section{INTRODUCTION}

A careful study of any book dealing with linear fracture mechanics (Broek) shows that much experimental data cannot be explained satisfactory in such a restricted theoretical framework. Among these data are two important phenomena: the stable growth of mode I cracks under monotonic loading and the thickness effect. Considering fracture to be closely related to the constitutive equations chosen for the material and given that elasticity is unable to explain either of the two above phenomena, we undertook this study by means of plasticity (Prager's linear kinematic hardening rule). The main advantage of such a law is its simplicity. It depends on only two parameters: a yield stress $\sigma_{0}$ and a hardening ratio a $=E_{l} / E$. This rule has the advantage of allowing a smooth transition from purely plastic behavior $(a=0)$ to purely elastic behavior $a=1)$. This is important since a fracture concept in elastoplasticity is not valid unless it can ultimately account for what occurs in material with very low ductility, i.e. brittle fracture.

\section{PREVIOUS RESULTS}

As the analytical study of a crack tip neighborhood is very complex in the elastoplastic case, we investigated the problem through numerical simulation. Such a method is not always simple due to the careful precautions that have to be taken in interpreting the results obtained.

First, we studied the case of a steadily growing crack, focusing on the dissipated energies that can be computed (Anquez, 1985). It appears that in a forced crack length increment $\Delta a$, under constant external loading, the overall dissipated energy $\Delta D_{R}$ is the sum of a decohesion energy $\Delta D_{e l}$ located at the crack tip and a dissipated plastic bulk energy $\Delta D_{p}$. Approximations of the rates of dissipated energies are obtained by dividing the energy increments by $\Delta a$ :

$$
G_{R}^{\Delta a}=G_{e l}^{\Delta a}+G_{p}^{\Delta a}
$$
In this equation, superscript $\Delta a$ points out the fact that each term is mesh size dependent. A study of numerical convergence shows that as $\Delta a$ approaches zero, $G_{R}{ }^{\Delta a}$ approaches a value close to ${ }^{2}$ which could be obtained in the pure elasticity case $\left(K={ }^{2}=\right.$ in the plane stress case) and $G_{l} l$ approaches zero. This brings us during crack growth. 
Despite this, we performed numerical simulations of stable crack growth under the meng loading: having found that, for a given mesh (chle crack growth und elements near state (i.e., stress and strain tensor components) in length $\Delta a$ ) mechanical quantities tip depended on one parameter only, at any step, (i. a i tip node whenever a cho interrelated), we undertook simulations by releasing a defined a priori. Each chosen characteristic parameter reached a critical valu characterized for ent a load increment dissipated, then a tip node by $\Delta K$-during which the bulk plastic $\Delta D M$ w critical value; during this last prece when the characteristic parameter reached pergy $\Delta D_{R}$ was dissipated.

The essential result obtained by means of numerical simulations was th

$\Delta D_{M}+\Delta D_{R}$ divided by $\Delta a$ remained quasical simulations was that the sum simulation. This result is confirmed by a quasi-constant at any step during a we can write:

$$
\frac{\partial D}{\partial K} \cdot \frac{d K}{d a}+\frac{\partial D}{\partial a}=\frac{d D}{d a}=G_{\mathrm{c}}
$$
We must point out here that such a result comes from numerical analysis and that
it could not be easily established by thermodynamics.

Careful analysis of the numerical results obtained during simulation a write (when small scale yielding hypotheses ared during simulation allows us to

$$
\begin{aligned}
& \frac{\partial D}{\partial a} \simeq G_{c}\left(\frac{K}{K_{c}}\right)^{2 \gamma} \\
& \frac{\partial D}{\partial K}=\frac{\Gamma}{o_{0}^{2} E} K^{3}
\end{aligned}
$$

where $Y$ and $\Gamma$ are functions of the hardening ratio a. When $a=0$ (perfect
plasticity), $Y=1$ and $\Gamma=\Gamma_{0}$ so wehave:

$$
\frac{d K^{2}}{d a}=2 \frac{o_{o}^{2}}{\Gamma_{o}}\left(\frac{K_{c}^{2}}{K^{2}}-1\right)
$$
When a approaches 1 (pure elasticity), $y$ approaches 0.5 and $\Gamma$ approaches 0.0 , so we
obtain the following well-known result: $K=K_{c}$

These results were obtained in the plane stress case. Similar results are also obtained in the plane strain case. The question of the thickness effect then arises: if a given critical value $G_{c}$ is $G$ is near $\varphi$ canly not the same because, as mentioned above, in the stea plane stress parameter will no could be calculated in pure elasticity. So taking $G_{c}$ as a mate case, paratet thickness effect. We therefore must investigaterial

\section{THICKNESS EFFECT: A SUGGESTION} The starting point of our argument is the following: if a crack can grow in plasticity
without any recourse to decohesion, this is because its growth is due only to the
accumulation of infinitesimal plastic slips at the tip.
Thus, special attention has to be devoted to the slip lines which can be found by looking for the lines of maximum plastic shear strain (bisectors of the main axes of the incremental plastic strain tensor).

As an example, we present the slip lines obtained for the same a value $(a=0.1)$ and the same $K$ value, in the plane stress case for a stationary crack (Fig. 1) and for a steadily growing crack (Fig. 2), and in the plane strain case for a stationary crack (Fig. 3) and for a steadily growing crack (Fig. 4).

Three zones can be distinguished in these above cases

Zone 1, located ahead of the crack, where the slip lines do not reach the crack tip but cross the crack propagation axis at an angle of approximately $n / 4$. This zone, whose relative size increases as a decreases in plane stress, is negligible in plane strain.

Zone 2 , where the slip lines reach the crack tip forming an angle less than or equal to $n / 2$ with respect to the crack propagation axis.

Zone 3 where the slip lines reach the crack tip from behind, forming an angle greater than $n / 2$ with respect to the crack propagation axis.

As only the slips at the tip (which result from what takes place all along the corresponding slip line) which are at an angle less than or equal to $n / 2$ can combine w produce a crack tip advance, we concentrated our attention on zone 2 .

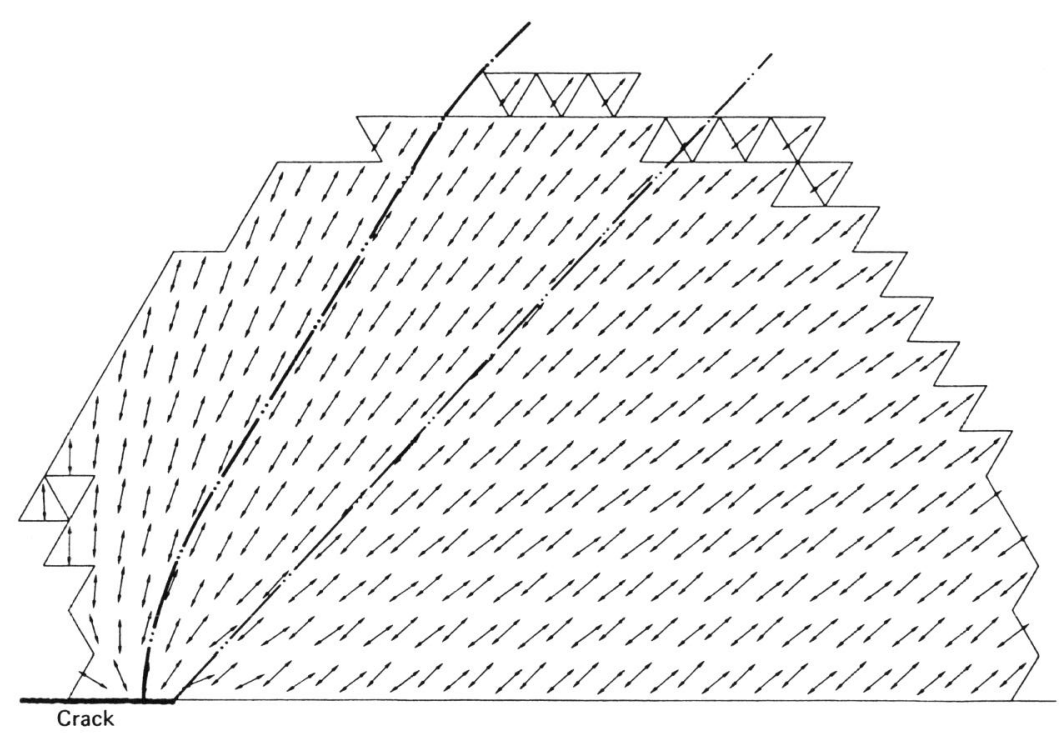

Fig. 1 - Plane stress. Stationary crack. Slip line field at $K_{D}$ 


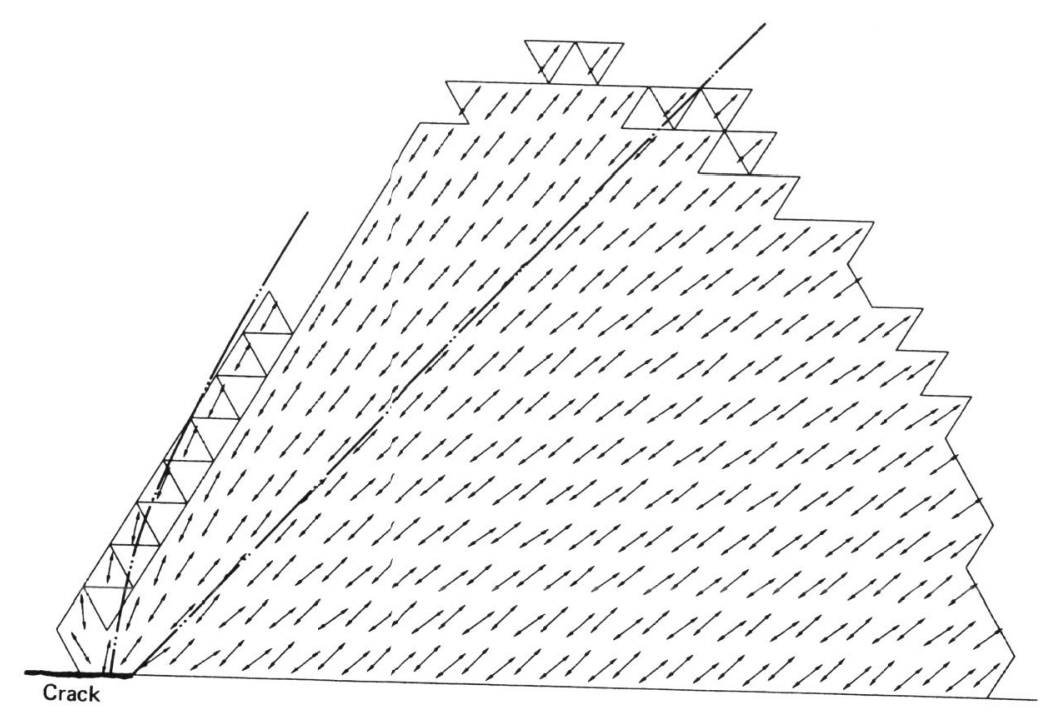

Fig. 2 - Plane stress. Steadily growing crack. Slip line field at $K_{D}$.

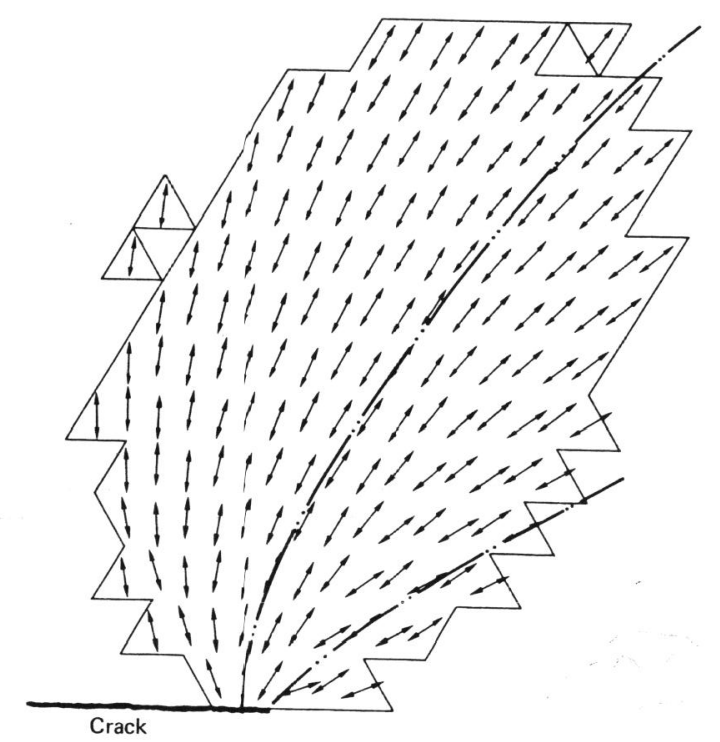

Fig. 3 - Plane strin. Stationary crack. Slip line field at $K_{D}$.

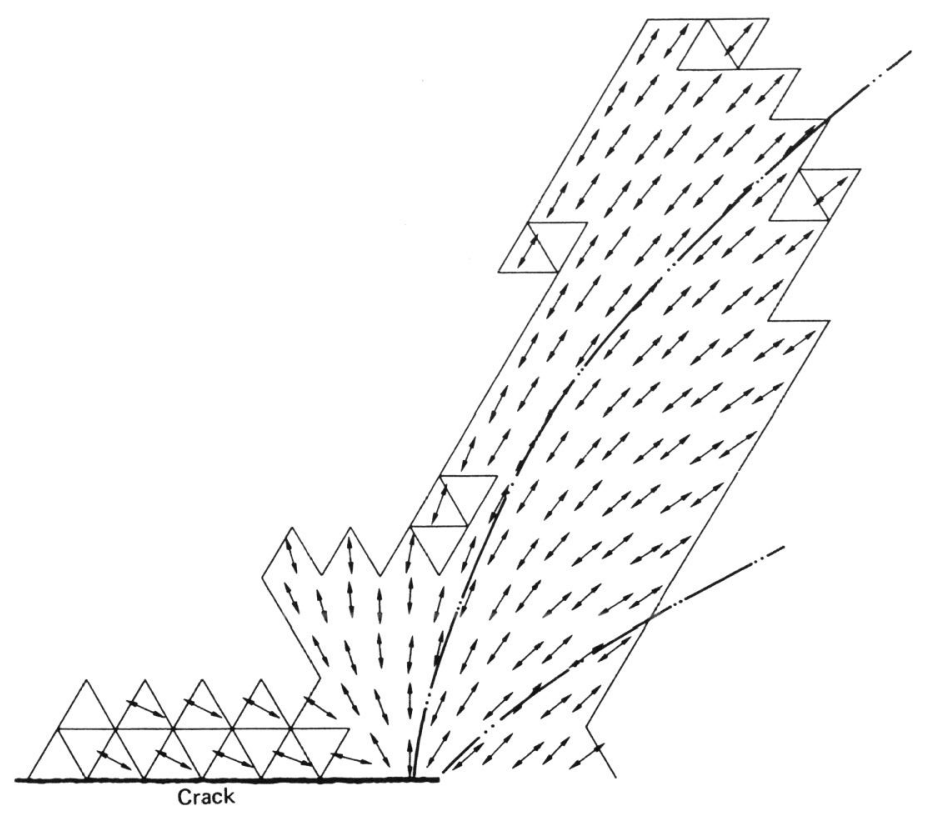

Fig. 4 - Plane strain. Steadily growing crack. Slip line field at $K_{D}$.

A good measure of the plastic activity in this zone, which causes the slip concentration at the tip, is the plastic energy increment $\Delta d$ dissipated either during the load increment $\Delta K$, the crack being fixed, or during a crack propagation increment $\Delta a$, the load being fixed.

The last step is to determine as well as possible the spatial limits of zone 2 and to calculate the ratio $\eta$ of $\Delta d$ (increment of energy dissipated in this zone) to $\Delta D$ (increment of bulk energy dissipated) in the two following cases: loading without crack growth and crack growth under constant load (in plane stress and in plane strain, for different values of the hardening ratio a).

The results obtained are shown in Fig. 5 and Fig. 6.

The essential feature observed is that the ratios $\eta_{C P}$ and $\eta_{D P}$ have almost the same value in the stationary and the steady state case. So we can write:

$$
\frac{\partial d}{\partial K}=\eta \frac{\partial D}{\partial K} \quad \frac{\partial d}{\partial a}=\eta \frac{\partial D}{\partial a}
$$

Multiplying equation (2) by $\eta$ then gives:

$$
\frac{\partial d}{\partial K} \cdot \frac{d K}{d a}+\frac{\partial d}{\partial a}=\eta G_{c}=g_{c}
$$

So there exists a critical $g_{c}$ defined in zone 2 . This energy release rate is more meaningful than $G_{c}$ as far as the crack propagation is concerned.

We now assume that this value is intrinsic to the material; so we decide that $g_{c}$ is the same in plane strain and in plane stress. 


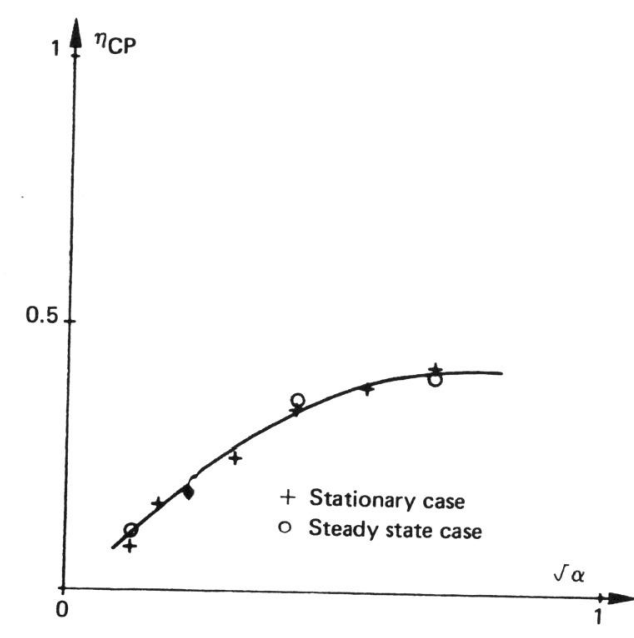

Fig. 5 - Plane stress. Evolution of the ratio $\eta$ of the useful plastic energy increment to the overall plastic energy versus $\sqrt{ } \alpha$ ( $\alpha=E_{t} / E$ being the hardening ratio).

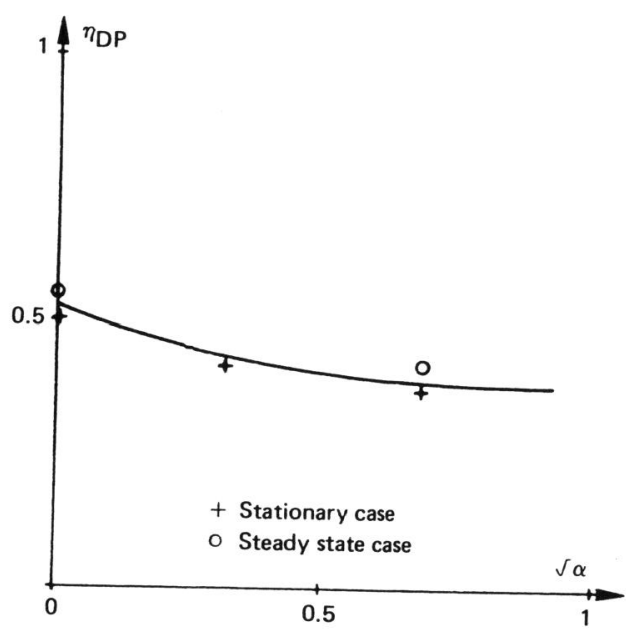

Fig. 6 - Plane strain. Evolution of $\eta$ versus $\sqrt{\alpha}$

Given this, suppose that we know $K_{I_{C}}$ for a given material. Then:

$$
\operatorname{Cy}_{c}^{D P}=\frac{\left(1-v^{2}\right)}{E} K_{l}^{2}
$$

Having pointed out that $G$ and $\varphi_{\text {have }}$ approximately the same value, we could write:

$$
G_{c}^{D P} \simeq \frac{\left(1-v^{2}\right)}{E} K_{I}^{2}
$$

Knowing the material, we can find its hardening ratio a and consequently (see Fig. 6) its appropriate $\eta$ value in plane strain. So:

$$
g_{\mathrm{c}}=\eta_{D P} G_{\mathrm{c}}^{D P} \simeq \eta_{D P} \frac{\left(1-\mathrm{v}^{2}\right)}{E} K_{I_{C}}^{2}
$$

Then to determine $K_{1 c}$, whose ratio to $K_{I_{C}}$ is a characteristic of the thickness effect, we write:

$$
g_{c}=\eta_{C P} G_{c}^{C P} \simeq \eta_{C P} \frac{K_{1 c}^{2}}{E}
$$

And finally:

$$
\frac{K_{1 c}^{2}}{K_{I}^{2}} \simeq\left(1-v^{2}\right) \frac{{ }^{\eta_{D P}}}{{ }^{\eta_{C P}}}
$$

The evolution of the ratio $K_{1} / K_{I_{C}}$ versus a (Fig. 7) shows that the theory suggested seems to be able to properly describe the thickness effect.

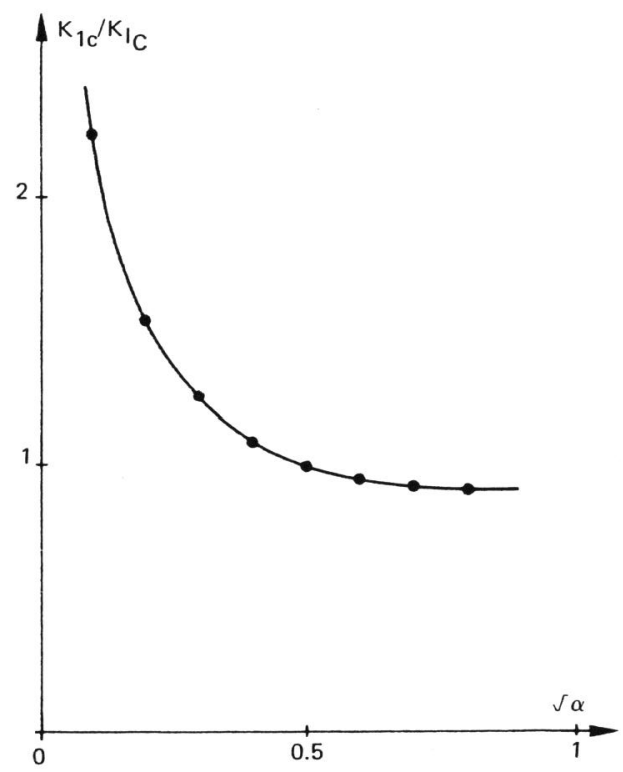

Fig. 7 - Thickness effect versus $\sqrt{ } \alpha$. 


\section{CONCLUSION}

The final result presented in Fig. 7 is encouraging but requires further

The first interest of $g_{c}$ is its physical meaning which allows us to consider it a small scale yielding).

The second interest of $g_{c}$ is that it is independent of the mesh size, provided the iently refined.

Finally, the use of $g_{c}$ allows us to use the same "simple" theory to consider stable crack growth, the thickness effect and also fatigue crack growth, since we have found (As well as perh) that in this last case, an energy balance similar to (2) can be growth as well a REFERENCES

Anquez, L. (1985). Etude numérique de la rupture ductile en élastoplasticité, tendances actuelles en calcul de structure. Bastia, Editions Pluralis.

Anquez, L. (1988). Contribution of Numerical Modelling to Fatigue Crack Growt Society. 3rd International Spring Meeting of the French Metallurgical

Broek, D.i.

Internationentary Engineering Fracture Mechanics. Noordhoff International Publishing Leyden. 